\title{
The Prepared Reflex: Behavioral and Subjective Flanker Interference Effects
}

\author{
Pin-Wei Chen ${ }^{1}$, Tiffany K. Jantz ${ }^{2} \&$ Ezequiel Morsella ${ }^{1,3}$ \\ ${ }^{1}$ Department of Psychology, San Francisco State University, California, USA \\ ${ }^{2}$ Department of Psychology, University of Michigan, Ann Arbor, Michigan, USA \\ ${ }^{3}$ Department of Neurology, University of California, San Francisco, California, USA \\ Correspondence: Ezequiel Morsella, Department of Psychology, San Francisco State University, 1600 Holloway \\ Avenue, EP 301, San Francisco, California 94132-4168, USA. E-mail: morsella@sfsu.edu
}

Received: September 1, 2014

Accepted: September $172014 \quad$ Online Published: November 10, 2014

doi:10.5539/ijps.v6n4p1

URL: http://dx.doi.org/10.5539/ijps.v6n4p1

\begin{abstract}
One can easily learn to associate a motor response to a given sensory stimulus. This linking of stimuli to responses ("S-R links," for short) may be learned through verbal instruction or through extensive training. The former has been characterized as something akin to the acquisition of a "prepared reflex." Recently, it has been demonstrated that, in the flanker paradigm, S-R links acquired through prepared reflexes can yield the interference effects found with the traditional versions of this task, which normally include training. In a fully within-subjects paradigm, we replicated this current research and extended it, by including (a) contrasts between all traditional flanker conditions (including response interference and perceptual interference) and (b) trial-by-trial subjective measures of performance (i.e., "urges to err"). The behavioral and subjective effects found with prepared reflexes resembled those found following normal S-R link acquisition. The theoretical implications of this finding are discussed.
\end{abstract}

Keywords: action control, automaticity, perception-and-action, prepared reflex, stimulus-response link, working memory

\section{The Prepared Reflex: Behavioral and Subjective Flanker Interference Effects}

It is trivial to demonstrate that humans can quickly learn to associate a motor response to a given sensory stimulus. The phenomenon, which occurs both in everyday life, in educational settings, and in countless laboratory experiments, is often instantiated by a simple instruction, such as "press the square button when the light is green" or "turn the knob when you hear a click." The learning process for such associations is often quick and effortless. This striking ability to link stimuli to responses ("S-R links," for short) has been a primary object of study in the history of psychology. Till today, there is debate regarding the mechanisms that lead to such rapid acquisition of S-R links (cf., Gallistel \& Gibbon, 2001; Hommel, Müsseler, Aschersleben, \& Prinz, 2001; Mitchell, De Houwer, \& Lovibond, 2009; Morsella, Riddle, \& Bargh, 2009).

Independent of this debate about the mechanisms underlying the rapid, single-trial acquisition of S-R links, investigators have attempted to illuminate the different ways in which these associations could be learned. This line of research examines the distinct properties of different forms of S-R acquisition (e.g., through verbal instruction or extensive practice). For example, an S-R link may be learned only through verbal instruction or through extensive practice in which the indicated response is emitted toward the stimulus repeatedly over the course of scores of trials. The latter form of S-R acquisition may lead to automatized responses (Puttemans, Wenderoth, \& Swinnen, 2005).

The acquisition of an S-R link through mere verbal instruction, involving only one trial and without any training, has been characterized as something akin to the acquisition of a "prepared reflex" or "psychic reflex" (Ach, 1905; Cohen-Kdoshay \& Meiran, 2009; Exner, 1879; Gollwitzer, 1999; Hommel, 2000; Woodworth, 1939). These terms reflect that the effects of this form of knowledge acquisition resemble, remarkably, those of automatized S-R links acquired through training. It has been proposed that, in the prepared reflex, working memory is taxed (Cohen-Kdoshay \& Meiran, 2007; Cohen-Kdoshay \& Meiran, 2009; Meiran \& Cohen-Kdoshay, 2012), because the rule regarding the stimulus-response contingency is actively held in mind. (Working memory has been defined 
as a temporary, capacity-limited storage system under attentional control that is used to intentionally hold and manipulate information in mind; Baddeley, 1986, 2007.) Researchers today have begun to accumulate data regarding the similar properties of S-R acquisition from the prepared reflex and from traditional stimulus-response training (Cohen-Kdoshay \& Meiran, 2007, 2009), in which the S-R link is acquired over the course of several trials (Eriksen \& Eriksen, 1974). To examine these similarities, researchers have employed response interference paradigms such as the classic flanker task (Eriksen \& Eriksen, 1974), our next topic of discussion. We will return to the topic of the prepared reflex after discussing this behavioral paradigm.

\subsection{The Flanker Task}

In the flanker paradigm (Eriksen \& Eriksen, 1974), one must respond to a visual target and disregard flanking "distractors." In one variant of the paradigm (Eriksen \& Schultz, 1979), during flanker training, participants are first trained in 30 or more trials to press one button with one finger when presented with the letter S or $\mathrm{M}$ and to press another button with another finger when presented with the letter $\mathrm{P}$ or $\mathrm{H}$. After training, which involves the acquisition of these four S-R links, participants are then instructed to respond to the letter presented in the center of an array and to disregard the flanking letters (the "distractors"). Interference (e.g., increased response times [RTs] and error rates) is stronger when distractors and targets are associated with different actions (response interference $[\mathrm{RI}])$, as in the case of PPSPP, than when distractors and targets look different but are associated with the same response (perceptual interference [PI]; Eriksen, 1995; Wilson et al., 2009), as in the case of MMSMM. (Shortest RTs occur in the Identical condition, in which the flankers are identical to targets [e.g., SS $\underline{S} S S$, target underscored].)

Unlike the original flanker task (Eriksen \& Eriksen, 1974), which included only the Identical and RI conditions, the Eriksen and Schultz (1979) version of the flanker task includes a PI condition that permits one to contrast the interference effects arising from perceptual-level and response-level stages of processing (van Veen, Cohen, Botvinick, Stenger, \& Carter, 2001). Consistent with the patterns of results found by Eriksen and Schultz (1979), van Veen et al. (2001) found that RI led to a mean RT of $624 \mathrm{~ms}(S D=79)$ and PI led to mean of $565 \mathrm{~ms}(S D=74)$, with the difference between RI and PI being 59 ms. Similarly, in Wilson et al., 2009), RI led to a mean RT of $734.01 \mathrm{~ms}(S E=31.78)$, and PI led to mean of $672.51 \mathrm{~ms}(S E=33.52)$, with the difference between RI and PI being $61.5 \mathrm{~ms}$.

Evidence from psychophysiological studies suggests that RI stems from the automatic, "stimulus-triggered" activation of action plans by visual distractors (DeSoto, Fabiani, Geary, \& Gratton, 2001). Specifically, studies revealed that, in RI, competition involves simultaneous activation of the brain areas associated with both the target-related and distractor-related responses (DeSoto et al., 2001; Mattler, 2005). In addition, functional brain imaging research has shown that RI and PI have distinct neural signatures, with RI involving more activations in frontal cortex, including frontal regions associated with motor processes (van Veen \& Carter, 2006; van Veen et al., 2001).

In subjective variants of the flanker task, conscious perturbations such as trial-by-trial ratings of "urges to err" are strongest in the RI condition (Wilson et al., 2009). To obtain these subjective effects, participants are asked after each trial to introspect and rate on an 8-point scale "how strong the urge was to make a mistake" (cf., Gray et al., 2009; Wilson et al., 2009). In this scale, 1 signifies "no urge" and 8 signifies "strong urge." The observation that urges to err are strongest in the RI condition is consistent with the hypothesis that incompatible skeletomotor plans must trigger detectable changes in subjective experience (see quantitative review of additional evidence from a plethora of interference paradigms in Morsella, Berger, \& Krieger, 2011). Such research on the subjective aspects of performance has revealed insights that may not be learned from behavioral measures alone (Etkin, Prater, Hoeft, Menon, \& Schatzberg, 2010; Morsella et al., 2011).

In assessing the similarities between the two forms of S-R acquisition, researchers have demonstrated that, in the flanker task, the prepared reflex can yield the RI effect that is found in flanker paradigms that include flanker training (Cohen-Kdoshay \& Meiran, 2007, 2009). This is quite a remarkable effect. However, other aspects of the prepared reflex remain unexplored. For example, no research to date on the prepared reflex has provided data regarding the additional contrasts featured in the flanker paradigm (i.e., those involving the three interference conditions of Identical, PI, and RI). Second, no research has examined the trial-by-trial subjective effects associated with the prepared reflex. Do these subjective effects resemble what is found in subjective variants of the traditional flanker task, which includes training?

Our aim was to build upon and replicate previous research (Cohen-Kdoshay \& Meiran, 2007, 2009) while providing new data that address these unexplored issues. Such data would further illuminate the similarities between the two distinct forms of S-R acquisition. To our knowledge, the present project is the first investigation 
to examine these similarities while taking into consideration all conditions of the flanker task and trial-by-trial, subjective effects. In addition, we performed a separate analysis to examine whether these similarities are detectable during the earliest of flanker trials following the acquisition of the S-R link through the mechanisms of the prepared reflex.

\subsection{Overview and Predictions}

In a within-subjects design, participants completed two blocks of flanker trials under two conditions, each reflecting a different form of S-R acquisition: a Prepared Reflex condition and a traditional, Training-Based condition. Each condition consisted of a block of trials. Block order was fully counterbalanced across participants. In the Training-Based condition, participants completed a flanker training session (trials $=128)$ before beginning the critical block of trials. This condition is likely to involve automatic responding (MacLeod \& Dunbar, 1988; Proctor \& Lu, 1999; Puttemans et al., 2005). In the Prepared Reflex condition, participants completed the flanker trials without any training. Hence, to perform the task, participants presumably held the instructions in mind, taxing working memory, as theorized in previous research (Cohen-Kdoshay \& Meiran, 2009; Meiran \& Cohen-Kdoshay, 2012; Oberauer \& Hein, 2012). Thus, this condition is likely to involve working memory to a greater extent than the Training-Based condition. In addition, in this condition there should be less automatic responding than in the Training-Based condition. In our experiment, participants were faced with three types of flanker trials: Identical, PI, and RI. After each trial, participants were asked to rate how strong the urge to err was on that trial. Based on previous studies (Cohen-Kdoshay \& Meiran, 2007, 2009; Meiran \& Cohen-Kdoshay, 2012), we hypothesized that the Prepared Reflex condition should yield behavioral and subjective flanker effects similar to those found in the Training-Based condition.

\section{Method}

\subsection{Participants}

San Francisco State University undergraduates $(n=68$, female $=47)$ participated for course credit.

\subsection{Stimuli and Apparatus}

Stimuli were presented on an Apple iMac computer monitor $(50.8 \mathrm{~cm})$ with a viewing distance of approximately $48 \mathrm{~cm}$. Stimulus presentation and data recording were controlled by PsyScope software (Cohen et al., 1993). All letter stimuli $\left(1 \mathrm{~cm}^{2}\right)$ were white, presented in the center, on a black background.

\subsection{Procedures}

During training for the Training-Based condition (trials = 128), participants learned to associate two of the four letters (e.g., S and M) with pressing "4" on the keypad and the other two letters (e.g., P and H) with pressing "5" on the keypad. Participants were given specific key associations, such as "Use your index finger to press 4 for $S$ or $M$," or "Use your middle finger to press 5 for P or H." Participants were instructed to rest their hand on the keyboard and maintain contact with the keys at all times during the study. During the training trials, a fixation $(+)$ appeared $(500 \mathrm{~ms})$ in the center of the screen to direct the participant's attention. Following the fixation, a letter appeared in the center of the screen until the participant pressed the correct button. During the critical trials, participants were given the same instructions as were provided before the training trials.

During critical trials ( $n=64: 32$ Identical, 16 PI, and 16 RI trials), participants responded to a target letter that was flanked by four distractor letters (e.g., SSMSS). There was a larger proportion of Identical trials compared to each of the other interference conditions (PI or RI) in order to avoid habituation effects to the presence of the dissimilar flanking distractors (Gratton, Coles, \& Donchin, 1992; Lehle \& Hübner, 2008). Participants were informed that, after each trial, they would be asked to rate their urge to err on a scale from 1 to 8 , in which 1 represented "almost no urge" and 8 represented "extremely strong urge." Participants were instructed to input their responses by typing with their left hand. The associations of key presses to target stimuli were fully counter-balanced across participants. The pairing of targets to distractors in the flanker array was only partially counter-balanced due to the fact that the PI and RI conditions cannot be fully counterbalanced.

Each critical trial proceeded as follows. "Ready" appeared in the center of the screen along with a written reminder about the key associations. Participants pressed a button to initiate the trial. A fixation (+) appeared (500 ms) in the center of the screen to direct the participant's attention. This was followed by a 5-letter flanker array, which would also appear in the center of the screen $(\sim 4.5 \mathrm{~cm}$ wide $\mathrm{x} 1 \mathrm{~cm}$ high). Participants were instructed to respond as quickly and accurately as possible to the letter in the center of the array and to disregard the letters flanking the central letter. The array remained on the screen until the participant responded. Following the response, participants were asked, "How strong was your urge to make a mistake?" Participants responded by inputting a number from 1 to 8 . 
The Prepared Reflex condition was identical to the Training-Based condition, with two exceptions. First, this condition did not have training trials. Second, it was comprised of different letter stimuli. The presentation of these sets of stimuli were fully counterbalanced across participants. For this condition, before commencing the critical trials, the experimenter reiterated the instructions with the participant to make sure that each participant received the same amount of verbal instruction in both S-R acquisition conditions.

After completing the experiment, participants filled out a funneled debriefing form (following the procedures detailed in Bargh \& Chartrand, 2000) to assess participants' knowledge of the hypothesis at hand. Specifically, participants were asked: "What do you think the purpose of this experiment was?", "What do you think this experiment was trying to study?", "Did you have any strategy and/or goal in completing this experiment?", "Was there anything that interfered with your performance on the task?", "Have you ever participated in a study in which you respond to a letter in the center of other letters? For example, AACAA. (If YES, continue to the next two questions.)", "If so, what was the name of the study? How long ago did you participate in the study?", and "What were the letters you were trained on? Please list them below."

The data from a total of four participants were removed from analysis. Two of these participants had previous experiences in studies involving the flanker task. One participant was unable to complete the task within the allotted time; another participant erred on more than $20 \%$ of the trials. For RT analysis, RTs below $200 \mathrm{~ms}$ and above 2 s were excluded from analysis (based on Gray et al., 2009; Wilson et al., 2009; van Veen et al., 2001; Woodworth \& Schlosberg, 1954). The RT data from trials in which participants made an erroneous response were excluded from analysis. Regarding the urge to err ratings, typographical errors on the part of participants led to the loss of $27(0.66 \%)$ of 4,096 trials.

\section{Results}

\subsection{Response Times Analysis}

Descriptive statistics for this and all other dependent measures are presented in Table 1. A fully within-subjects ANOVA on the RTs revealed a main effect of S-R Acquisition (Training-Based condition versus Prepared Reflex condition), $F(1,63)=19.10, p<.001$, in which RTs were longer in the Prepared Reflex condition than in the Training-Based condition, as one would expect. There was also a main effect of Interference, $F(2,126)=30.10, p$ $<.001$, resembling the pattern of RTs found in the flanker paradigm. Planned comparisons revealed that, within the Training-Based condition, the contrasts between the Interference conditions were all significant $(t \mathrm{~s}>2.5, p \mathrm{~s}<.05)$, and that, within the Prepared Reflex condition, all the contrasts between Interference conditions were significant except for that between Identical and PI $(p=.19)$. There was no interaction between the factors S-R Acquisition and Interference, $F(2,126)=0.16, p=.86$. For this dependent measure (and for other dependent measures), there was no main effect $(p>.05)$ of the order in which participants received the S-R Acquisition conditions. As mentioned above, the order sequence was fully counterbalanced across subjects.

Table 1. Descriptive statistics as a function of condition

\begin{tabular}{|c|c|c|c|c|c|c|c|c|c|c|}
\hline & \multicolumn{2}{|c|}{$\underline{\text { Conditions }}$} & \multicolumn{2}{|c|}{ Error Rate } & \multicolumn{2}{|c|}{$\underline{\mathrm{RT}}$} & \multicolumn{2}{|c|}{$\underline{\text { Urge }}$} & \multicolumn{2}{|c|}{$\underline{\text { RT Data Trimmed }}$} \\
\hline & $\frac{\underline{\mathrm{S}-\mathrm{R}}}{\text { Acquisition }}$ & $\frac{\text { Interference }}{\underline{\text { Condition }}}$ & Mean & $\underline{\mathrm{SE}}$ & $\underline{\text { Mean }}$ & $\underline{\mathrm{SE}}$ & Mean & $\underline{\mathrm{SE}}$ & $\underline{\text { Mean }}$ & $\underline{\mathrm{SE}}$ \\
\hline \multirow{9}{*}{$\begin{array}{l}\text { Full } \\
\text { Experiment }\end{array}$} & & Grand & 0.05 & 0.06 & 873.68 & 24.41 & 2.49 & 0.11 & 0.11 & 0.01 \\
\hline & $\begin{array}{l}\text { Training-Bas } \\
\underline{\text { ed }}\end{array}$ & $\begin{array}{l}\text { Across } \\
\text { conditions }\end{array}$ & 0.05 & 0.01 & 825.23 & 23.36 & 2.37 & 0.12 & 0.09 & 0.01 \\
\hline & & Identical & 0.05 & 0.01 & 785.91 & 25.66 & 2.09 & 0.11 & 0.09 & 0.01 \\
\hline & & PI & 0.02 & 0.01 & 815.82 & 24.08 & 2.27 & 0.13 & 0.06 & 0.01 \\
\hline & & RI & 0.08 & 0.01 & 873.96 & 24.98 & 2.76 & 0.17 & 0.13 & 0.02 \\
\hline & $\begin{array}{l}\text { Prepared } \\
\text { Reflex }\end{array}$ & $\begin{array}{l}\text { Across } \\
\text { conditions }\end{array}$ & 0.05 & 0.01 & 922.14 & 29.87 & 2.61 & 0.12 & 0.13 & 0.02 \\
\hline & & Identical & 0.04 & 0.01 & 883.69 & 29.39 & 2.29 & 0.12 & 0.10 & 0.01 \\
\hline & & PI & 0.04 & 0.01 & 905.98 & 32.05 & 2.53 & 0.13 & 0.11 & 0.02 \\
\hline & & RI & 0.07 & 0.01 & 976.74 & 33.30 & 3.01 & 0.16 & 0.18 & 0.02 \\
\hline
\end{tabular}




\begin{tabular}{|c|c|c|c|c|c|c|c|c|c|c|}
\hline & $\begin{array}{l}\text { Across } \\
\text { Conditions }\end{array}$ & Identical & 0.05 & 0.01 & 834.80 & 19.91 & 2.19 & 0.08 & 0.09 & 0.01 \\
\hline & & PI & 0.03 & 0.01 & 860.90 & 20.36 & 2.40 & 0.09 & 0.08 & 0.01 \\
\hline & & RI & 0.08 & 0.01 & 925.35 & 21.23 & 2.89 & 0.11 & 0.16 & 0.02 \\
\hline \multirow[t]{12}{*}{$\underline{\text { First } 16 \text { trials }}$} & & Grand & 0.07 & 0.01 & 948.86 & 26.00 & 2.64 & 0.13 & 0.16 & 0.02 \\
\hline & $\begin{array}{l}\text { Training-Bas } \\
\text { ed }\end{array}$ & $\begin{array}{l}\text { Across } \\
\text { conditions }\end{array}$ & 0.07 & 0.02 & 865.16 & 36.48 & 2.50 & 0.18 & 0.11 & 0.03 \\
\hline & & Identical & 0.07 & 0.02 & 847.13 & 37.11 & 2.22 & 0.20 & 0.10 & 0.03 \\
\hline & & PI & 0.03 & 0.02 & 857.20 & 33.11 & 2.41 & 0.22 & 0.05 & 0.03 \\
\hline & & RI & 0.11 & 0.03 & 891.15 & 31.96 & 2.88 & 0.23 & 0.17 & 0.05 \\
\hline & $\begin{array}{l}\text { Prepared } \\
\underline{\text { Reflex }}\end{array}$ & $\begin{array}{l}\text { Across } \\
\text { conditions }\end{array}$ & 0.07 & 0.02 & 1032.56 & 37.01 & 2.77 & 0.18 & 0.21 & 0.03 \\
\hline & & Identical & 0.06 & 0.02 & 996.03 & 45.45 & 2.45 & 0.16 & 0.17 & 0.03 \\
\hline & & PI & 0.05 & 0.02 & 1059.61 & 50.55 & 2.72 & 0.21 & 0.18 & 0.03 \\
\hline & & RI & 0.10 & 0.03 & 1080.23 & 50.49 & 3.14 & 0.20 & 0.29 & 0.05 \\
\hline & $\begin{array}{l}\text { Across } \\
\text { Conditions }\end{array}$ & Identical & 0.06 & 0.01 & 912.35 & 28.30 & 2.33 & 0.13 & 0.14 & 0.02 \\
\hline & & PI & 0.04 & 0.01 & 948.53 & 29.02 & 2.57 & 0.15 & 0.11 & 0.02 \\
\hline & & RI & 0.11 & 0.02 & 985.69 & 29.68 & 3.01 & 0.15 & 0.23 & 0.03 \\
\hline
\end{tabular}

$P I=$ perceptual interference; $R I=$ response interference; "RT Data Trimmed" includes the removal of response times below $200 \mathrm{~ms}$ or above $2 \mathrm{~s}$ and the exclusion of response times from trials in which participants erred.

\subsection{Error Rate Analysis}

A fully within-subjects ANOVA on the raw error rates revealed no main effect of the factor S-R Acquisition, $F(1$, $63)=0.04, p=.84$, and a main effect of Interference, $F(2,126)=12.97, p<.001$. Planned contrasts revealed that, for the Prepared Reflex condition, the contrasts between Interference conditions were significant $(t \mathrm{~s}>2.2, p \mathrm{~s}<.05)$ except for that between Identical and PI $(p=.89)$. Within the Training-Based condition, the only nonsignificant contrast was between Identical and RI $(p=.08)$. Analysis on arcsine transformations of the data also revealed a similar pattern of results. (Arcsine transformations are often used to statistically normalize data that are in the form of proportions.) In this analysis, there was no main effect of S-R Acquisition, $F(1,63)=0.19, p=.66$. However, there was a main effect of the factor Interference, $F(2,126)=12.32, p<.001$. Planned contrasts revealed that, for the Training-Based condition, all contrasts between Interference conditions were significant $(p \mathrm{~s}<.05)$ except for that between Identical and RI $(p=.56)$. The planned contrasts revealed that, for the Prepared Reflex condition, all contrasts were nonsignificant $(p \mathrm{~s}>.16)$ except that between PI and RI $(p<.05)$. There was no interaction between the factors of S-R Acquisition and Interference on error rates, $F(2,126)=1.15, p=.32$.

\subsection{Subjective Effects: Urges}

A within-subjects ANOVA on the subjective, urge to err data (Table 1) revealed a main effect of S-R Acquisition, $F(1,63)=7.74, p=.007$, in which urges to err tended to be lower for the trained participants, as one would expect. There was no interaction between S-R Acquisition and Interference, $F(2,126)=.095, p=.89$. However, the analysis did reveal the predicted effect of Interference, $F(2,126)=31.43, p<.001$. Planned comparisons revealed that, within the Training-Based condition, all of the contrasts between Identical, PI, and RI were significant $(t \mathrm{~s}>$ $2.2, p \mathrm{~s}<.05)$. A similar pattern of Interference results was found in the Prepared Reflex condition $(t \mathrm{~s}>2.8, p \mathrm{~s}$ $<.01)$.

\subsection{Supplementary Analysis: Subjective Data Associated with Working Memory}

Through both piloting and information provided informally by some participants during the debriefing stage of our study, we learned of the possibility that, during the test trials, participants' consciousness might be occupied, not just with urges, but with the rehearsal of the instructions of the task at hand (e.g., rehearsal of the sensorimotor contingencies). Such a possibility is consistent with the hypothesis that, in the prepared reflex, working memory is used to hold stimulus-response contingencies in mind (Meiran \& Cohen-Kdoshay, 2012). The possibility is also 
consistent with the more general observation that information in working memory influences the contents of consciousness (Baddeley, 2007; Jantz, Tomory, Gazzaley, \& Morsella, 2013; Jantz et al., 2014).

To investigate this possibility more formally, we collected data from a separate group of participants $(n=33)$ who performed a task that was similar to ours except that, during each trial, (a) there was a 7-second delay preceding the appearance of the flanker stimulus (based on Gazzaley, Cooney, Rissman, \& D'Esposito, 2005), and (b), during this delay, participants could by button press indicate whenever they experienced visual or auditory mental imagery about the letters, the buttons, the association of the letters with the buttons, or anything else that could help them perform the task (see details of procedures in Appendix A). This technique involving the reporting of imagery was based on the methods of Tomory et al. (2013) and Jantz et al. (2014). As expected and consisted with the informal reports obtained during piloting, participants reported the occurrence of task-related imagery during the 7-second delay. Across conditions, the mean imagery rate per delay was 0.93 ( $S E=.18$, participants' means ranged from 0 to 3.05), a rate that is significantly above zero, $t(27)=5.28, p<.001$ (see details about results in Appendix A).

\subsection{Supplementary Analysis: Initial Trials}

One may propose that the flanker effect in the Prepared Reflex condition resulted, not from S-R contingencies being actively held in working memory, but from participants' responding to targets during the initial critical trials, which could result in a form of flanker training. However, a flanker RT effect can be found in an analysis of just the first 16 (fully counterbalanced) critical trials of this condition of our principal experiment, $F(2,60)=3.92, p=.025$, $\eta_{p}{ }^{2}=.12$. (See Table 1 for complete descriptive statistics of this subset of the data.) A similar pattern of results from the first 16 trials was found in a mixed design ANOVA in which S-R Acquisition was a between-subjects factor and Interference was a within-subjects factor. In this analysis, there was a main effect of Interference, $F(2$, $122)=5.43, p=.006$, and of S-R Acquisition, $F(1,61)=10.36, p=.002$. There was no interaction between the two factors, $F(2,122)=1.04, p=.355$.

This finding suggests that the effect found in the Prepared Reflex condition is not simply an artifact of participants' prolonged exposure to trials during the critical block. Interestingly, the effect of Interference on urges to err was also significant for the first 16 trials of the Prepared Reflex condition, $F(2,62)=5.90, p=.005, \eta_{p}{ }^{2}=.16$. A similar pattern of results from the first 16 trials was found in a mixed design ANOVA in which urges to err was the dependent measure, S-R Acquisition was a between-subjects factor, and Interference was a within-subjects factor. In this analysis, there was a main effect of Interference, $F(2,124)=14.80, p<.001$, and no effect of S-R Acquisition, $F(1,62)=1.12, p=.294$. There was no interaction between the two factors, $F(2,124)=.046, p=.954$. Complementing these urge to err data, a mixed ANOVA on arcsine transformations of the error rates revealed a main effect of Interference, $F(2,124)=3.45, p=.034$, no main effect of S-R Acquisition, $F(1,62)=.003, p=.955$, and no interaction between the two factors, $F(2,124)=.254, p=.776$. It should be noted that the pattern of results for error rates did not resemble that of the traditional flanker paradigm (see Table 1). This outcome could reflect that errors are infrequent, and only a few trials (i.e., $n=16$ ) were sampled in this analysis. Nevertheless, RI did induce more errors than Identical.

Faced with this additional, corroboratory evidence stemming from the first 16 trials, one could argue that the RT and subjective effects found during the first (fully counterbalanced) mini-block of trials of the Prepared Reflex condition reflected, not the effect of a prepared reflex, but the flanker training that could have transpired across those very 16 trials. To address this potential criticism, we examined participants' performance on only the first two trials of the Prepared Reflex condition. This was an informal and exploratory analysis, for several reasons. First, it was post-hoc and the subset of only two trials could not provide data about all the three conditions; at most, it could provide data only about two conditions per participant. Second, because the order of presentation of the interference conditions was random, for this analysis, some participants experienced only one condition (e.g., two RI trials) during the first two trials. Third, the first trial of any experiment is likely to generate an erroneous response, as the participant is not as familiarized with the task as he or she is on subsequent trials. Hence, unlike in our formal analysis, and to minimize data loss from this small subset of trials, we included in this analysis the raw RT data, including RT data from trials in which participants made an erroneous response.

Despite these limitations, if one pools together all the first and second trials of all the participants who performed the Prepared Reflex condition first, and then groups the trials (total $=64)$ by Interference condition, one still finds that the mean RTs follow the pattern found with the classic flanker task: Identical $(M=1,719.52, S E=179.84)$, PI $(M=1,789.00, S E=232.49)$, and RI $(M=2,121.20, S E=329.88)$. The urge to err data follow the same pattern: Identical $(M=2.85, S E=0.33)$, PI $(M=3.46, S E=0.64)$, and RI $(M=4.10, S E=0.48)$. Future research could more systematically investigate participants' performance on the first trials of the Prepared Reflex condition. In addition, 
future investigations could assess whether similar effects are found in an experiment in which S-R Acquisition is held as a between-subjects factor. In the present study, to diminish carry-over effects from incidental training, the order of presentation of the different forms of S-R Acquisition was counterbalanced across participants. Future research could rule out such artifacts by having S-R Acquisition be a pure, between-subjects factor.

\section{General Discussion}

Building on previous research (Cohen-Kdoshay \& Meiran, 2009; Meiran \& Cohen-Kdoshay, 2012) demonstrating the strikingly similar properties between S-R links acquired through training or through verbal instruction alone (as in the case of the prepared reflex), we demonstrated in a fully within-subjects design that action sets for the flanker task, when acquired as a prepared reflex, yield behavioral and subjective effects that are similar to those obtained from traditional flanker training. Our paradigm extends previous research (Cohen-Kdoshay \& Meiran, 2009; Meiran \& Cohen-Kdoshay, 2012) by contrasting all three traditional flanker conditions and by having trial-by-trial subjective measures of performance (i.e., "urges to err"), all in a within-subjects design.

Analysis of the RTs revealed that both forms of S-R link acquisition yield a similar pattern of interference effects. This pattern is also found when examining only the first mini-block of experimental trials, which suggests that the RT pattern found in the Prepared Reflex condition did not arise from an incidental form of flanker training during the critical trials. It is worth noting that, in the Prepared Reflex condition, the contrast between Identical and PI failed to reach significance $(p=.19)$. Whether this null result reflects something interesting about cognitive processing could be a topic of further research.

Unlike in previous studies, we also examined the subjective effects associated with each kind of S-R acquisition. Subjective measures can reveal aspects of processing that might not be evident through behavioral measures alone (e.g., Gray et al., 2013; Molapour, Berger, \& Morsella, 2011). In our experiment, for both forms of S-R acquisition, all of the contrasts between Identical, PI, and RI were significant. The observation that RI yielded the strongest urge to err, both within the Training-Based condition and the Prepared Reflex condition, is consistent with the hypothesis that incompatible skeletomotor plans must trigger detectable changes in subjective experience (Morsella, 2005; Morsella et al., 2011).

Our paradigm inherits the limitations of both the flanker task and paradigms involving introspection. Regarding the latter, it is challenging to verify what participants were introspecting at the moment that they were making their judgment about the urge to err. Self-reports are far from infallible, even if they occur just seconds after the relevant conscious experience (Block, 2007). Moreover, one cannot rule out that judgments were based on self-observations involving RT performance, accuracy, or on folk beliefs regarding how one should comport oneself in an experiment about response interference (see treatment of this issue in Wilson et al., 2009). Despite these limitations, our subjective effects were systematic and replicated the patterns of results found with more extensive experimentation using the flanker task (Morsella et al., 2011; Wilson et al., 2009).

Regarding the flanker task, one limitation inherent in all versions of this paradigm (Coles, Gratton, Bashore, Eriksen, \& Donchin, 1985; Eriksen \& Eriksen, 1974; Eriksen \& Schultz, 1979; van Veen et al., 2001) is that response interference still features some perceptual interference, possibly rendering it more complicated than the latter. Unfortunately, a flanker-like paradigm that can induce response interference without also invoking perceptual interference has yet to be developed. Hypothetically, this could be instantiated by having targets and distractors be perceptually identical but somehow cue different responses, if such a scenario is possible.

Nevertheless, as mentioned above, there is evidence that response interference is qualitatively distinct from perceptual interference and that its behavioral and subjective effects are not simply the outcome of increased difficulty (DeSoto et al., 2001; Gray et al., 2013; Mattler, 2005; van Veen \& Carter, 2006; van Veen et al., 2001). To illuminate this and other issues, future research using the present paradigm could examine the similarities between the neural correlates of both forms of S-R link acquisition (cf., Puttemans et al., 2005). Future research may also provide additional evidence for the involvement of working memory in the peculiar Prepared Reflex condition (see evidence in Meiran \& Cohen-Kdoshay, 2012). Our experiment was not designed to examine directly this aspect of processing. In addition, as discussed above, future investigations could also assess whether the kind of prepared reflex effect we detected arises in a study in which S-R Acquisition is a pure between-subjects factor. In such a study, if flanker effects still are detectable during the first trials of a condition preceded by no form of flanker training, then this would serve as strong evidence for a prepared reflex mechanism.

Despite all the shortcomings, it is worth noting that this examination of the two forms of S-R acquisition was embedded within a paradigm (the flanker task) that has stood the test of time and has come to be one of the most reliable paradigms in the study of cognitive control and interference. In addition, the subjective effects replicate what has been found previously (Molapour et al., 2011; Morsella et al., 2011; Morsella et al., 2009a, 2009b). 
It is a curious fact that, at this stage of understanding, there is no obvious property in behavioral performance (or even in trial-by-trial subjective reports) that can be used to discriminate between the two forms of S-R link acquisition. This is noteworthy because the two forms of acquisition do indeed feel very different to the actor. It seems that, if such a difference is found, the marker will be associated with working memory performance (Meiran $\&$ Cohen-Kdoshay, 2012). Unlike automaticity-based performance, such a capability includes conscious rehearsal of memoranda (Johnson \& Johnson, 2009), which was apparent in our supplemental data collection. It is our hope that, by building on our research, future investigtions will further isolate such a potential marker.

\section{Acknowledgments}

This research was supported by the Center for Human Culture and Behavior at San Francisco State University.

\section{References}

Ach, N. (1905/1951). Determining tendencies: Awareness. In D. Rapaport (Ed.), Organization and pathology of thought (pp. 15-38). New York: Columbia University Press. (Original work published in 1905).

Baddeley, A. D. (1986). Working memory. Oxford, England: Oxford University Press.

Baddeley, A. D. (2007). Working memory, thought and action. Oxford, UK: Oxford University Press. http://dx.doi.org/10.1093/acprof:oso/9780198528012.001.0001

Bargh, J. A., \& Chartrand, T. L. (2000). The mind in the middle: A practical guide to priming and automaticity research. In H. T. Reis, \& C. M. Judd (Eds.), Handbook of research methods in social and personality psychology (pp. 253-285). Cambridge, England: Cambridge University Press.

Block, N. (2007). Consciousness, accessibility, and the mesh between psychology and neuroscience. Behavioral and Brain Sciences, 30, 481-548. http://dx.doi.org/10.1017/S0140525X07002786

Cohen, J. D., MacWhinney, B., Flatt, M., \& Provost, J. (1993). PsyScope: A new graphic interactive environment for designing psychology experiments. Behavior Research Methods, Instruments, \& Computers, 25, 257-271. http://dx.doi.org/10.3758/BF03204507

Cohen-Kdoshay, O., \& Meiran, N. (2007). The representation of instructions in working memory leads to autonomous response activation: Evidence from the first trials in the flanker paradigm. The Quarterly Journal of Experimental Psychology, 60, 1140-1154.

Cohen-Kdoshay, O., \& Meiran, N. (2009). The representation of instructions operates like a prepared reflex: Flanker compatibility effects found in the first trial following S-R instructions. Experimental Psychology, 56, 128-133. http://dx.doi.org/10.1027/1618-3169.56.2.128

Coles, M. G. H., Gratton, G., Bashore, T. R., Eriksen, C. W., \& Donchin, E. (1985). A psychophysiological investigation of the continuous flow model of human information processing. Journal of Experimental Psychology: Human Perception and Performance, 11, 529-553. http://dx.doi.org/10.1037/0096-1523.11.5.529

DeSoto, M. C., Fabiani, M., Geary, D. C., \& Gratton, G. (2001). When in doubt, do it both ways: Brain evidence of the simultaneous activation of conflicting responses in a spatial Stroop task. Journal of Cognitive Neuroscience, 13, 523-536. http://dx.doi.org/10.1162/08989290152001934

Eriksen, C. W. (1995). The flanker task and response competition: A useful tool for investigating a variety of cognitive problems. Visual Cognition, 2, 101-118. http://dx.doi.org/10.1080/13506289508401726

Eriksen, B. A., \& Eriksen, C. W. (1974). Effects of noise letters upon the identification of a target letter in a nonsearch task. Perception and Psychophysics, 16, 143-149. http://dx.doi.org/10.3758/BF03203267

Eriksen, C. W., \& Schultz, D. W. (1979). Information processing in visual search: A continuous flow conception $\begin{array}{llll}\text { and experimental results. Perception and Psychophysics, 25, 249-263. } & \text {. }\end{array}$ http://dx.doi.org/10.3758/BF03198804

Etkin, A., Prater, K., Hoeft, F., Menon, V., \& Schatzberg, A. (2010). Failure of anterior cingulate activation and connectivity with the amygdala during implicit regulation of emotional processing in generalized anxiety disorder. American Journal of Psychiatry, 167, 545-554. http://dx.doi.org/10.1176/appi.ajp.2009.09070931

Exner, S. (1879). Physiologie der Grosshirnrinde. In L. Hermann (Ed.), Handbuch der Physiologie (Vol. 2, pp. 189-350). Leipzig, Germany: Vogel.

Gallistel, C. R., \& Gibbon, J. (2001). Computational versus associative models of simple conditioning. Current Directions in Psychological Science, 10, 146-150. http://dx.doi.org/10.1111/1467-8721.00136 
Gazzaley, A., Cooney, J. W., Rissman, J., \& D’Esposito, M. (2005). Top-down suppression deficit underlies working memory impairment in normal aging. Nature Neuroscience, 8, 1298-1300. http://dx.doi.org/10.1038/nn1543

Gollwitzer, P. M. (1999). Implementation intentions: Strong effects of simple plans. American Psychologist, 54, 493-503. http://dx.doi.org/10.1037/0003-066X.54.7.493

Gratton, G., Coles, M. G., \& Donchin, E. (1992). Optimizing the use of information: Strategic control of activation of responses. Journal of Experimental Psychology: General, 121, 480-506. http://dx.doi.org/10.1037/0096-3445.121.4.480

Gray, J. R., Bargh, J. A., \& Morsella, E. (2013). Neural correlates of the essence of conscious conflict: FMRI of sustaining incompatible intentions. Experimental Brain Research, 229, 453-456. http://dx.doi.org/10.1007/s00221-013-3566-5

Hommel, B. (2000). The prepared reflex: Automaticity and control in stimulus-response translation. In S. Monsell, \& J. Driver (eds.), Control of cognitive processes: Attention and performance XVIII (pp. 247-273). Cambridge, MA: MIT Press.

Hommel, B., Müsseler, J., Aschersleben, G., \& Prinz, W. (2001). The theory of event coding: A framework for perception and action planning. Behavioral and Brain Sciences, 24, 849-937. http://dx.doi.org/10.1017/S0140525X01000103

Jantz, T. K., Tomory, J. J., Gazzaley, A., \& Morsella, E. (2013). Subjective aspects of action control for delayed actions: Action-related imagery. Journal of Mental Imagery, 37, 21-48.

Jantz, T. K., Tomory, J. J., Merrick, C., Cooper, S., Gazzaley, A., \& Morsella, E. (2014). Subjective aspects of working memory performance: Memoranda-related imagery. Consciousness and Cognition, 25, 88-100. http://dx.doi.org/10.1016/j.concog.2014.01.013

Johnson, M. R., \& Johnson, M. K. (2009). Toward characterizing the neural correlates of component processes of cognition. In F. Roesler, C. Ranganath, B. Roeder, \& R. H. Kluwe (Eds.), Neuroimaging of human memory: Linking cognitive processes to neural systems (pp. 169-194). New York: Oxford University Press.

Lehle, C., \& Hübner, R. (2008). On-the-fly adaptation of selectivity in the flanker task. Psychonomic Bulletin \& Review, 15, 814-818. http://dx.doi.org/10.3758/PBR.15.4.814

MacLeod, C. M., \& Dunbar, K. (1988). Training and Stroop-like interference: Evidence for a continuum of automaticity. Journal of Experimental Psychology: Learning, Memory, and Cognition, 14, 126-135. http://dx.doi.org/10.1037/0278-7393.14.1.126

Mattler, U. (2005). Flanker effects on motor output and the late-level response activation hypothesis. The Quarterly Journal of Experimental Psychology, 58A, 577-601. http://dx.doi.org/10.1080/02724980443000089

Meiran, N., \& Cohen-Kdoshay, O. (2012). Working memory load but not multitasking eliminates the prepared reflex: further evidence from the adapted flanker paradigm. Acta Psychologica, 139, 309-313. http://dx.doi.org/10.1016/j.actpsy.2011.12.008

Meiran, N., \& Cohen-Kdoshay, O. (2009). The representation of instructions operates like a prepared reflex: Flanker compatibility effects found in first trial following S-R instructions. Experimental Psychology, 56, 128-133. http://dx.doi.org/10.1027/1618-3169.56.2.128

Mitchell, C. J., De Houwer, J., \& Lovibond, P. F. (2009). The propositional nature of human associative learning. Behavioral and Brain Sciences, 32, 183-198. http://dx.doi.org/10.1017/S0140525X09000855

Molapour, T., Berger, C. C., \& Morsella, E. (2011). Did I read or did I name? Process blindness from congruent processing 'outputs.' Consciousness and Cognition, 20, 1776-1780. http://dx.doi.org/10.1016/j.concog.2011.03.004

Morsella, E. (2005). The function of phenomenal states: Supramodular interaction theory. Psychological Review, 112, 1000-1021. http://dx.doi.org/10.1037/0033-295X.112.4.1000

Morsella, E., Berger, C. C., \& Krieger, S. C. (2011). Cognitive and neural components of the phenomenology of agency. Neurocase, 17, 209-230. http://dx.doi.org/10.1080/13554794.2010.504727

Morsella, E., Gray, J. R., Krieger, S. C., \& Bargh, J. A. (2009). The essence of conscious conflict: Subjective effects of sustaining incompatible intentions. Emotion, 9, 717-728. http://dx.doi.org/10.1037/a0017121 
Morsella, E., Krieger, S. C., \& Bargh, J. A. (2009). The function of consciousness: Why skeletal muscles are "voluntary" muscles. In. E. Morsella, J. A. Bargh, \& P. M. Gollwitzer (Eds.), Oxford handbook of human action (pp. 625-634). New York: Oxford University Press.

Morsella, E., Riddle, T. A., \& Bargh, J. A. (2009). Undermining the foundations: Questioning the basic notions of associationism and mental representations. Behavioral and Brain Sciences, 32, 218-219. http://dx.doi.org/10.1017/S0140525X09001071

Morsella, E., Wilson, L. E., Berger, C. C., Honhongva, M., Gazzaley, A., \& Bargh, J. A. (2009). Subjective aspects of cognitive control at different stages of processing. Attention, Perception, \& Psychophysics, 71, 1807-1824. http://dx.doi.org/10.3758/APP.71.8.1807

Oberauer, K., \& Hein, L. (2012). Attention to information in working memory. Current Directions in Psychological Science, 21, 164-169. http://dx.doi.org/10.1177/0963721412444727

Proctor, R. W., \& Lu, C. H. (1999). Processing irrelevant location information: Practice and transfer effects in choice-reaction tasks. Memory \& Cognition, 27, 63-77. http://dx.doi.org/10.3758/BF03201214

Puttemans, V., Wenderoth, N., \& Swinnen, S. P. (2005). Changes in brain activation during the acquisition of a multifrequency bimanual coordination task: From the cognitive stage to advanced levels of automaticity. Journal of Neuroscience, 25, 4270-4278. http://dx.doi.org/10.1523/JNEUROSCI.3866-04.2005

van Veen, V., \& Carter, C. S. (2006). Conflict and cognitive control in the brain. Current Directions in Psychological Science, 5, 237-240. http://dx.doi.org/10.1111/j.1467-8721.2006.00443.x

van Veen, V., Cohen, J. D., Botvinick, M. M., Stenger, V. A., \& Carter, C. C. (2001). Anterior cingulate cortex, conflict monitoring, and levels of processing. Neuroimage, 14, 1302-1308. http://dx.doi.org/10.1006/nimg.2001.0923

Woodworth, R. S. (1939). The cause of a voluntary movement. Psychological issues: Selected papers of Robert S. Woodworth. New York: Columbia University Press.

Woodworth, R. S., \& Schlosberg, H. (1954). Experimental psychology (2nd ed.). New York: Holt, Rinehart \& Winston.

\section{Appendix A}

\section{Supplemental Data Collection}

For our supplemental data collection, all stimuli and apparatus were identical to those of our principal study with three exceptions: (1) participants used a button-box (ioLab Systems: UK) in order to both respond to target letters and report imagery, (2) participants' subjective urges to err were not collected, and (3) the letter $O$ was replaced by the letter $\mathrm{G}$ in order to avoid any artifacts from having the letters $\mathrm{O}$ and $\mathrm{K}$ being associated with the same button-press response. All responses were made on a button-box that consisted of eight colored buttons: six buttons on the top, one on the left side, and one on the right side. Participants were instructed to rest their right hand on the top of the button box and maintain contact in this way for the duration of the study. All letter stimuli (the set: $\mathrm{S}, \mathrm{M}, \mathrm{P}, \mathrm{H}$, and $\mathrm{G}, \mathrm{K}, \mathrm{F}, \mathrm{Z}$ ) were in white, 48-point, Helvetica font. All stimuli appeared in the center of a black background and were approximately $1 \mathrm{~cm}^{2}$ in size.

Based on Jantz et al. (2014), participants were given examples of mental imagery and what it means to have visual and auditory imagery. Regarding visual mental imagery, the onscreen instructions stated, "Take a moment to imagine what a tree looks like. Take a moment to imagine what a car looks like. You have just experienced an example of visual mental imagery." Regarding auditory mental imagery, participants read, "Without saying it aloud, take a moment to imagine what the word 'HOUSE' sounds like. Take a moment to imagine what the word 'FLOWER' sounds like. You have just experienced an example of auditory mental imagery." Participants were told that they would be asked to report about their mental imagery at a later time. Participants then completed a training block and a prepared reflex block. Block order was counterbalanced fully across participants. In the training block, participants were trained ( $n=128$ trials) on letter-to-button associations for one letter set (e.g., $\mathrm{S}$ and $\mathrm{M} ; \mathrm{P}$ and $\mathrm{H}$ ) before completing the critical trials $(n=64)$. As in our principal study, during training trials, participants learned to press one button with their right index finger when presented with either of two letters from a set (e.g., $\mathrm{S}$ and $\mathrm{M}$ ) and to press another button with their right middle finger when presented with either of two other letters from the same set (e.g., $\mathrm{P}$ and $\mathrm{H})$. 
For the critical trials, participants were instructed to press, during the delay, the left-side button on the button box with their left hand every time they experienced visual or auditory mental imagery about the letters, the buttons, the association of the letters with the buttons, or any mental imagery that would help them perform the task during the delay. The experimenter verbally reiterated the example of visual and auditory mental imagery provided at the beginning of the study and confirmed with participants that they understood the instructions. Following this reminder, the critical trials commenced. Critical trials transpired as follows. "Ready" appeared in the center of the screen with a reminder of the letter-to-button associations on the bottom of the screen. Participants pressed a button with their right small finger (i.e. pinky finger) to initiate the trial. A fixation $(+)$ appeared in the center of the screen $(7 \mathrm{~s})$. During this delay, participants used their left hand to pressed the left side-button to indicate that they had experienced mental imagery. Then a blank screen appeared $(500 \mathrm{~ms})$, followed by a 5-letter flanker array appeared in the center of the screen. Because of a software malfunction during data recording, the data from five participants were excluded from analysis. Imagery rates were comparable for the two conditions $\left(M_{\text {Prepared Reflex }}=\right.$ $\left.1.01, S E=.19 ; M_{\text {Training }}=0.84, S E=.18\right), t(27)=1.437, p=.16$.

\section{Copyrights}

Copyright for this article is retained by the author(s), with first publication rights granted to the journal.

This is an open-access article distributed under the terms and conditions of the Creative Commons Attribution license (http://creativecommons.org/licenses/by/3.0/). 